\title{
Hormone receptor status predicts the clinical outcome of human epidermal growth factor 2-positive metastatic breast cancer patients receiving trastuzumab therapy: a multicenter retrospective study
}

This article was published in the following Dove Press journal:

OncoTargets and Therapy

II November 2015

Number of times this article has been viewed

\author{
Yunchao Wang' \\ Tao Sun ${ }^{2}$ \\ Donggui Wan ${ }^{3}$ \\ Lijun Sheng ${ }^{4}$ \\ Wei $\mathrm{Li}^{5}$ \\ Huayun Zhu \\ Yanping $\mathrm{Li}^{\prime}$ \\ Janice $\mathrm{Lu}^{1,7}$
}

'Department of Breast Cancer, Beijing Shijitan Hospital, Capital Medical University, Beijing, ${ }^{2}$ Department of Oncology, Liaoning Cancer Hospital and Institute, Shenyang, ${ }^{3}$ Department of Oncology, China-Japan Friendship Hospital, Beijing, ${ }^{4}$ Department of Oncology, Shandong Cancer Hospital, Jinan, ${ }^{5}$ Department of Oncology, the First Affiliated Hospital of Nanjing Medical University, ${ }^{6}$ Department of Oncology, Jiangsu Cancer Hospital of Nanjing Medical University, Nanjing, People's Republic of China; ${ }^{7}$ Division of Hematology and Oncology, David Geffen School of Medicine, University of California, Los Angeles, CA, USA

Correspondence: Yanping Li; Janice Lu Department of Breast Cancer, Beijing Shijitan Hospital, Capital Medical University, 10 Tieyi Road, Haidian District, Beijing 100038, People's Republic of China

Tel +86 I53 01378638 ;

+8618618195136

Email lyp67I202@sina.cn;

mjlu@mednet.ucla.edu
Objectives: Trastuzumab, a humanized monoclonal antibody that binds human epidermal growth factor receptor 2 (HER2), dramatically improves the clinical outcomes of HER2positive breast cancer. Emerging evidence implied that the clinical behavior and sensitivity to targeted agents in HER2-positive breast cancer differed by hormone receptor (HR) status. The objective of this study was to determine the effect of the HR status on survival benefit of HER2-positive metastatic breast cancer when treated with anti-HER2-targeted therapy in People's Republic of China.

Methods: Metastatic breast cancer patients with HER2-positive diseases across six cancer centers in People's Republic of China were retrospectively analyzed in our study. Patients were classified into four groups according to HR/HER2 status and trastuzumab treatment: HER2+/ $\mathrm{HR}+$ patients with first-line trastuzumab treatment, HER2+/HR+ patients with no trastuzumab treatment, HER2+/HR - patients with first-line trastuzumab treatment, and HER2+/HR-patients with no trastuzumab treatment. Kaplan-Meier analysis, log-rank test, and multivariate analysis were performed during analysis.

Results: A total of 295 patients were included in the final analysis. The median overall survival was 30 months (95\% confidence interval: 27.521-32.479). Among patients with HER2+/ HR- disease, significant survival benefit was observed when treated with trastuzumab (30 vs 21 months, $P=0.000$ ). However, in patients with HER2+/HR+ disease, trastuzumab administration had a survival improvement trend but no significant statistical differences (36 vs 30 months, $P=0.258$ ). In the multivariate analysis, HR status was an independent predictor of overall survival and trastuzumab treatment had significantly decreased risk of death in HER $2+$ / HR- patients (hazard ratio $=0.330$ ).

Conclusion: HR status is an independent predictor of overall survival in HER2-positive metastatic breast cancer patients and patients with HER2+/HR- subtype might be associated with more survival benefits when treated with trastuzumab-based regimens.

Keywords: HER2-positive, metastatic breast cancer, hormone receptor status

\section{Background}

Human epidermal growth factor receptor type 2 (HER2) is overexpressed in 15\%-20\% of invasive breast cancers and predicts a poor prognosis prior to the era of HER2targeted therapy. ${ }^{1-5}$ Trastuzumab, a humanized monoclonal antibody that binds HER2, dramatically improves the clinical outcomes of HER2-positive breast cancer. ${ }^{6-11}$ 
Patients with HER2-positive breast cancer are more likely to develop metastases compared with HER2-negative breast cancer patients, and anti-HER2 agents such as trastuzumab in combination with chemotherapy are often a preferred first-line therapeutic option for these patients. ${ }^{6,12}$

Hormone receptors such as estrogen receptor (ER) and progesterone receptor (PR) also regulate breast tumor cell proliferation and are usually considered prognostic predictors of endocrine treatment. ${ }^{13}$ Initially, an inverse association was noted between HER2 positivity and hormonal receptors expression, but subsequently it was proved that up to $50 \%$ of the patients with HER2-positive tumors are also HR positive, ${ }^{14}$ although HR was expressed at lower levels compared with HER2-/HR+ tumors. ${ }^{15}$ It was thought that antiHER2 agents were effective in patients with HER2-positive disease, irrespective of the HR status. ${ }^{16}$ However, recent data have suggested that HER2+/HR+ and HER2+/HR- tumors are two different subtypes, with different prognoses in the absence of HER2 blockade therapy. ${ }^{17-19}$ Data from neoadjuvant and adjuvant settings also indicate that HR-positive and HR-negative tumors among HER2-positive breast cancer patients exhibit different response rates to treatment ${ }^{20}$ and relapse patterns. ${ }^{21,22}$ A large retrospective analysis showed that HER2-positive tumors with HR-negative expression exhibited greater lymphocytic infiltration, and higher HER2 intensity. ${ }^{23,24}$ Another study revealed that HER2 heterogeneity affected trastuzumab responses and survival in patients with HER2-positive metastatic breast cancer (MBC). ${ }^{25}$ Assessment of HR status helps to direct therapy for breast cancer patients. Before the HR testing guidelines were published, the threshold for positive HR status was controversial and the test's performance lacked consistency. ${ }^{26-28}$ HER2 test performance has improved since the publication of the guidelines, ${ }^{29}$ but there are still limited data on the influence of HR status on trastuzumab treatment benefits in HER2-positive MBC. In the current study, we retrospectively investigated the relationship of HR and HER2 status with trastuzumab treatment and its effect on outcomes of patients with MBC across multiple cancer centers in People's Republic of China.

\section{Patients and methods}

\section{Patients}

This retrospective study was conducted at six cancer centers across People's Republic of China, which included Beijing Shijitan Hospital, Liaoning Cancer Hospital and Institute, China-Japan Friendship Hospital, Shandong Cancer Hospital, the First Affiliated Hospital of Nanjing Medical University, and Jiangsu Cancer Hospital. Patients who met all the following criteria were included in the study: 1) a pathological diagnosis of adenocarcinoma of the breast; 2) at least 18 years of age; 3 ) immunohistochemical staining showed $3+$ intensity for the HER 2 protein $(>30 \%$ of invasive tumor cells with intense membrane staining) or amplification of the HER 2 gene on fluorescence in situ hybridization (ratio of HER2 to chromosome 17 centromere $\geq 2.0$ ); ${ }^{29}$ 4) pathologically and/or radiologically proven metastatic disease. Patients who met the criteria above but with one of the following conditions were excluded: 1) lack of definite ER and PR status; 2) received prior systemic therapy for metastatic disease; 3 ) received $<2$ cycles of chemotherapy as the first-line chemotherapy without computed tomography scan; 4) incomplete medical records and follow-up data; 5) other primary tumors.

Institutional review board approval was obtained from the Ethics Committee of Beijing Shijitan Hospital for our analyses, and the study was performed according to the Declaration of Helsinki.

\section{Therapeutic regimen}

The patients received trastuzumab at a loading dose of $8 \mathrm{mg} / \mathrm{kg}$ of body weight, followed by $6 \mathrm{mg} / \mathrm{kg}$ of body weight every 3 weeks, or a loading dose of $4 \mathrm{mg} / \mathrm{kg}$ of body weight, followed by $2 \mathrm{mg} / \mathrm{kg}$ of body weight weekly, either by itself or concurrent with chemotherapy.

\section{Data collection}

Individual patient data regarding baseline characteristics, histopathologic results at surgery, prior chemotherapy and endocrine therapy, first-line regimens, initial number of metastases and metastatic sites, date of disease progression, and death were extracted for this analysis from the electronic medical records at each institution. The follow-up data were collected from electronic medical records or by telephone interviews. The primary tumor was staged according to the seventh edition of the American Joint Committee on Cancer tumor-node-metastasis (TNM) classification. ${ }^{30}$ Positive HR status was defined as staining of $1 \%$ or more of either the ER or PR expression on immunohistochemical analysis.

\section{Follow-up study}

Median follow-up duration was calculated as the median observation time for the patients. The primary endpoint of the study was overall survival (OS) and the secondary endpoint was progression-free survival (PFS). OS was calculated from the first date of relapse (local, regional, or distant) to the date of death from any cause or the date of the last follow-up. 
PFS was calculated from the date of diagnosis of MBC until first disease progression after the initiation of treatment for MBC. The first disease progression was defined as local recurrence and/or distant metastasis that occurred after the initiation of treatment for MBC. Metastatic disease was diagnosed by clinical evaluations including imaging studies or biopsy, and disease progression detected by imaging and clinical examination.

\section{Statistical analysis}

Kaplan-Meier method was used to estimate OS and PFS and their corresponding 95\% confidence intervals (CIs). Patients without progression or death were censored at the last follow-up visit. Log-rank test was used for univariate analysis and variables with $P<0.20$ on univariate analysis and a clinically important variable (age) were included in the Cox proportion hazard regression model. For example, factors included in the multivariate analysis models on OS in HER2-positive MBC were age ( $\leq 50$ vs $>50$ years), pathological primary tumor TNM stage (I-III vs IV), HR status (positivity vs negativity), prior adjuvant endocrine therapy (receiving vs not receiving), disease-free survival interval ( $<24$ vs $\geq 24$ months), metastases site (non-visceral vs visceral metastases), initial number of metastases (single vs multiple metastases), trastuzumab treatment (with trastuzumab treatment vs without). Univariate and multivariate Cox regression analyses were used to generate unadjusted and adjusted HR and their corresponding 95\% CIs. All the statistical analyses were performed using the SPSS 17.0 statistical software program (SPSS Inc., Chicago, IL, USA) and $P<0.05$ was considered statistically significant.

\section{Results}

\section{Patient demographic and baseline characteristics}

The study flowchart is shown in Figure 1. Nine hundred and thirty-two patients were diagnosed with $\mathrm{MBC}$ at the six participating hospitals between January 1, 2009 and June 1, 2014. Four hundred and eleven (44.1\%) of them were positive for HER2 expression but 116 patients were excluded because

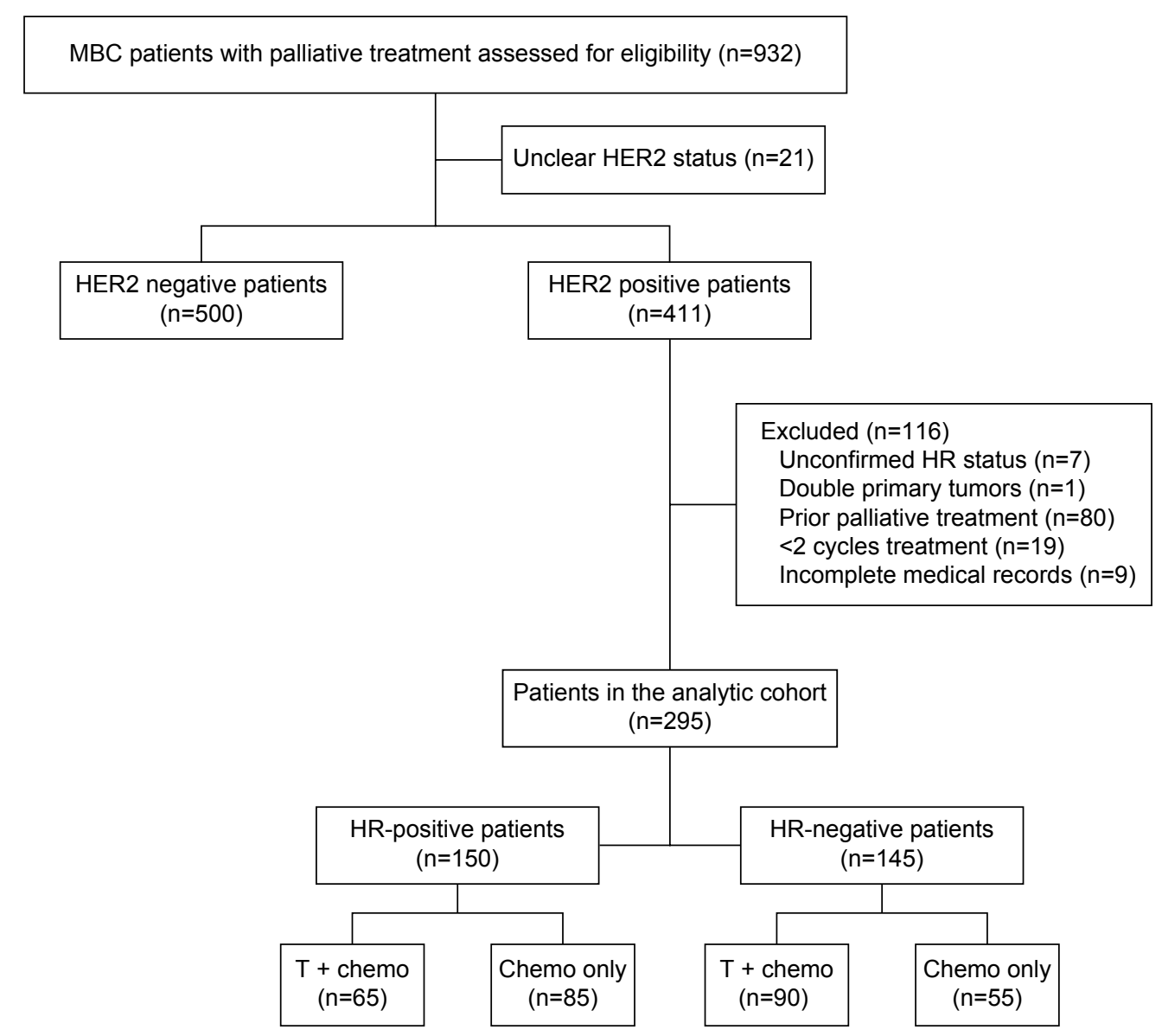

Figure I The study flowchart.

Abbreviations: chemo, chemotherapy; HER, human epidermal growth receptor; HR, hormone receptor; MBC, metastatic breast cancer; T, trastuzumab. 
of unclear HR status $(n=7)$, the presence of other primary tumor $(n=1)$, prior palliative treatment $(n=80),<2$ cycles of treatment $(n=19)$, or incomplete medical records $(n=9)$. Consequently, 295 patients were included in the final analysis. Their demographic and baseline characteristics are shown in Table 1. Their median age was 52 (range 26-80) years and slightly more than half (52.5\%) of them were premenopausal. Additionally, approximately half (50.8\%) of them were HR positive. Immunohistochemical staining showed 3+ intensity for the HER2 protein in 206 (69.8\%) patients. Approximately half of the women (52.5\%) received trastuzumab therapy and the median cycle of first-line trastuzumab-based therapy was

Table I Demographic and baseline characteristics of the study population

\begin{tabular}{|c|c|c|c|}
\hline Variables & $\begin{array}{l}\text { All } \\
\text { patients }\end{array}$ & $\begin{array}{l}\text { Trastuzumab } \\
\text { therapy }\end{array}$ & $\begin{array}{l}\text { No trastuzumab } \\
\text { therapy }\end{array}$ \\
\hline Patients, n (\%) & $295(100.0)$ & $155(52.5)$ & $140(47.5)$ \\
\hline \multicolumn{4}{|l|}{ Age, years } \\
\hline Median (range) & $52(26-80)$ & $52(26-77)$ & $52(30-80)$ \\
\hline$\leq 50$ years, $\mathrm{n}(\%)$ & $129(43.7)$ & $66(22.4)$ & $63(21.3)$ \\
\hline \multicolumn{4}{|l|}{ Postmenopausal status, n (\%) } \\
\hline Yes & $155(52.5)$ & $80(27.1)$ & $75(25.4)$ \\
\hline No & 140 (47.5) & $75(25.4)$ & $65(22.1)$ \\
\hline \multicolumn{4}{|l|}{ Histology, n (\%) } \\
\hline IDC & $286(96.9)$ & $152(5 \mid .5)$ & I $34(45.4)$ \\
\hline ILC & $9(3.1)$ & $3(1.0)$ & $6(2.1)$ \\
\hline \multicolumn{4}{|l|}{ Tumor grade, n (\%) } \\
\hline $\mathrm{I}-2$ & $94(31.9)$ & $57(19.3)$ & $37(12.6)$ \\
\hline 3 & $65(22.0)$ & $37(12.5)$ & $28(9.5)$ \\
\hline Unknown & $136(46.1)$ & $61(20.7)$ & $75(25.4)$ \\
\hline \multicolumn{4}{|l|}{ Lymph node invasion, $\mathrm{n}(\%)$} \\
\hline 0 & $79(26.8)$ & $34(I I .5)$ & $45(15.3)$ \\
\hline $1-3$ & $89(30.2)$ & 47 (I5.9) & $42(14.3)$ \\
\hline $4-9$ & $56(18.9)$ & $34(11.5)$ & $22(7.4)$ \\
\hline$>9$ & $71(24.1)$ & $40(13.6)$ & $31(10.5)$ \\
\hline \multicolumn{4}{|l|}{ TNM stage, $\mathrm{n}(\%)$} \\
\hline I-III & $267(90.5)$ & I 37 (46.4) & $130(44.1)$ \\
\hline IV & $28(9.5)$ & $18(6.1)$ & $10(3.4)$ \\
\hline \multicolumn{4}{|l|}{ HR status, n (\%) } \\
\hline Positive & $150(50.8)$ & $65(22.0)$ & $85(28.8)$ \\
\hline $\mathrm{ER}+/ \mathrm{PR}+$ & $102(34.6)$ & $38(12.9)$ & $64(21.7)$ \\
\hline $\mathrm{ER}+/ \mathrm{PR}-$ & $36(12.2)$ & $20(6.8)$ & $16(5.4)$ \\
\hline ER-/PR+ & $12(4.0)$ & $7(2.3)$ & $5(1.7)$ \\
\hline Negative & $145(49.2)$ & $90(30.5)$ & $55(18.7)$ \\
\hline \multicolumn{4}{|l|}{ HER2 evaluation, $n(\%)$} \\
\hline IHC intensity $3+$ & $206(69.8)$ & $93(31.5)$ & $113(38.3)$ \\
\hline IHC intensity $2+$ or I+, FISH amplification & $89(30.2)$ & $62(21.0)$ & $27(9.2)$ \\
\hline \multicolumn{4}{|l|}{ Prior adjuvant chemotherapy, n (\%) } \\
\hline Yes & $247(83.7)$ & $128(43.4)$ & $119(40.3)$ \\
\hline No & $48(16.3)$ & $27(9.1)$ & $21(7.2)$ \\
\hline \multicolumn{4}{|l|}{ Prior adjuvant endocrine therapy, $n(\%)$} \\
\hline Yes & $87(29.5)$ & $39(44.8)$ & $48(55.2)$ \\
\hline No & $208(70.5)$ & $116(39.3)$ & $92(31.2)$ \\
\hline \multicolumn{4}{|l|}{ Prior adjuvant targeted therapy, $\mathrm{n}(\%)$} \\
\hline Yes & $39(13.2)$ & $24(8.1)$ & $15(5.1)$ \\
\hline No & $256(86.8)$ & 131 (44.4) & $125(42.4)$ \\
\hline \multicolumn{4}{|l|}{ Disease-free survival interval, n (\%) } \\
\hline$<24$ months & $166(56.3)$ & $96(32.6)$ & $70(23.7)$ \\
\hline$\geq 24$ months & $129(43.7)$ & $59(20.0)$ & $70(23.7)$ \\
\hline \multicolumn{4}{|l|}{ Metastases, n (\%) } \\
\hline Visceral & $174(58.9)$ & $93(31.5)$ & $81(27.4)$ \\
\hline Multiple & $108(36.6)$ & $49(16.6)$ & $59(50.0)$ \\
\hline \multicolumn{4}{|l|}{ First-line chemotherapy, n (\%) } \\
\hline Taxane-based & I6I (54.6) & $79(26.8)$ & $82(27.8)$ \\
\hline Vinorelbine-based & $53(18.0)$ & 35 (II.9) & $18(6.1)$ \\
\hline Others & $81(27.4)$ & $41(13.9)$ & $40(13.5)$ \\
\hline
\end{tabular}

Abbreviations: ER, estrogen receptor; FISH, fluorescent in situ hybridization; HER2, human epidermal growth factor receptor 2; HR, hormone receptor; IDC, invasive ductal carcinoma; IHC, immunohistochemistry; ILC, invasive lobular carcinoma; PR, progesterone receptor; TNM, tumor-node-metastasis. 
6 (range 2-15). Women receiving trastuzumab therapy and not receiving trastuzumab therapy were comparable in demographic and baseline characteristics $(P>0.05$ in all).

\section{OS}

The median follow-up duration from first date of treatment for MBC patients was 34 (range 10-74) months. One hundred and fifty-four $(52.2 \%)$ deaths were recorded at the time of data analysis. The median OS for all women was 30 months (95\% CI: 27.521-32.479) (Figure 2A). HER2+/HR+ and
HER2+/HR - women receiving or not receiving trastuzumab therapy were comparable in demographic and baseline characteristics $(P>0.05)$ (Table 2$)$. Among patients with HER2+/HR- disease, patients receiving trastuzumab therapy showed markedly longer OS (median 30 months; 95\% CI: 25.557-34.443) than those not receiving trastuzumab therapy (median 21 months; 95\% CI: 13.904-28.096) $(P=0.000)$ (Figure 2B). However, in patients with HER2+/ $\mathrm{HR}+$ disease, trastuzumab administration showed longer OS (median 36 months; 95\% CI: 28.176-43.824) than those
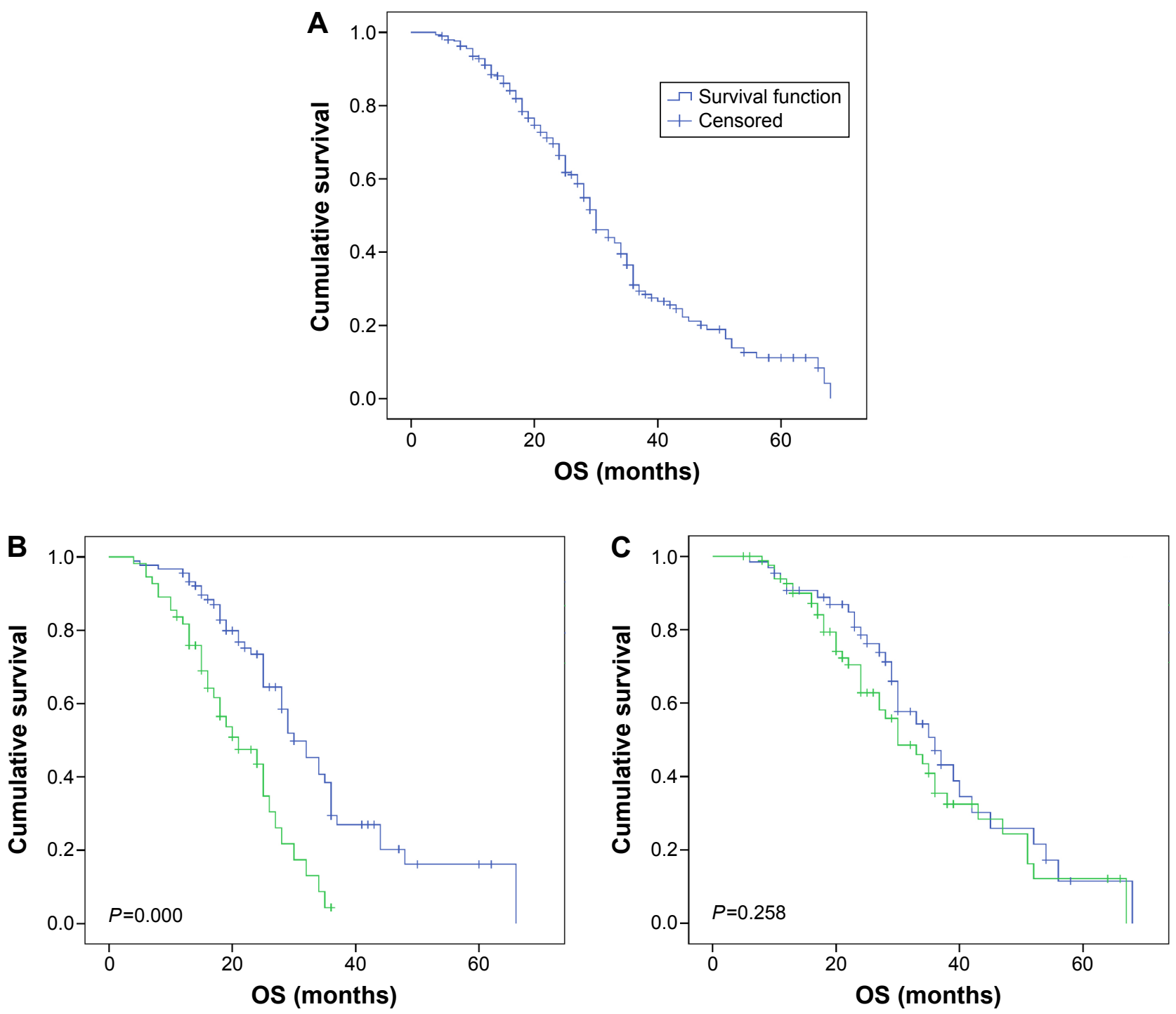

$$
\begin{array}{ll}
\text { Treatment } & \\
\neg \text { HER2+ with trastuzumab } & + \text { HER2+ with trastuzumab-censored } \\
\neg \text { HER2+ without trastuzumab } & + \text { HER2+ without trastuzumab-censored }
\end{array}
$$

Figure 2 Median overall survival (OS) regarding HR status and trastuzumab treatment.

Notes: (A) Survival curve for all patients; (B) survival curves for HR-negative patients regarding trastuzumab treatment; and (C) survival curves for HR-positive patients regarding trastuzumab treatment.

Abbreviations: HER2, human epidermal growth factor receptor 2; HR, hormone receptor; OS, overall survival. 
Table 2 Demographic and baseline characteristics of HR-positive and HR-negative MBC patients according to trastuzumab therapy

\begin{tabular}{|c|c|c|c|c|c|c|}
\hline \multirow[t]{3}{*}{ Variables } & \multirow{2}{*}{\multicolumn{2}{|c|}{$\begin{array}{l}\text { HR }+ \\
\text { Trastuzumab therapy }\end{array}$}} & \multirow[t]{3}{*}{$P$-value } & \multirow{2}{*}{\multicolumn{2}{|c|}{$\begin{array}{l}\text { HR- } \\
\text { Trastuzumab therapy }\end{array}$}} & \multirow[t]{3}{*}{$P$-value } \\
\hline & & & & & & \\
\hline & Yes & No & & Yes & No & \\
\hline $\mathrm{N}(\%)$ & $65(43.3)$ & $85(56.7)$ & & $90(62.1)$ & $55(37.9)$ & \\
\hline Age, years & & & 0.338 & & & $0.54 I$ \\
\hline Median (range) & $52(26-76)$ & $52(30-75)$ & & $52(29-77)$ & $53(36-80)$ & \\
\hline$\leq 50$ years, $\mathrm{n}(\%)$ & $27(4 I .5)$ & $42(49.4)$ & & $39(43.3)$ & $21(39.6)$ & \\
\hline Postmenopausal, n (\%) & & & 0.692 & & & 0.338 \\
\hline Yes & $35(53.8)$ & $43(50.6)$ & & $45(50.0)$ & $32(58.2)$ & \\
\hline Histology, n (\%) & & & 0.203 & & & 0.986 \\
\hline IDC & $65(100.0)$ & $80(94.1)$ & & $87(96.7)$ & $54(98.2)$ & \\
\hline ILC & $0(0.0)$ & $5(5.9)$ & & $3(3.3)$ & $\mathrm{I}(\mathrm{I} .8)$ & \\
\hline Tumor grade, n (\%) & & & 0.123 & & & 0.483 \\
\hline $1-2$ & $22(33.8)$ & $19(22.4)$ & & $35(38.9)$ & $18(32.7)$ & \\
\hline 3 & $17(26.2)$ & $18(21.1)$ & & $20(22.2)$ & $10(18.2)$ & \\
\hline Unknown & $26(40.0)$ & $48(56.5)$ & & $35(38.9)$ & $27(49.1)$ & \\
\hline Lymph node invasion, $\mathrm{n}(\%)$ & & & 0.262 & & & 0.520 \\
\hline 0 & $16(24.6)$ & $32(37.6)$ & & $18(20.0)$ & $13(23.6)$ & \\
\hline $1-3$ & $24(36.9)$ & $23(27.1)$ & & $23(25.6)$ & $19(34.5)$ & \\
\hline $4-9$ & $13(20.0)$ & $12(14.1)$ & & $21(23.3)$ & $10(18.3)$ & \\
\hline$>9$ & $12(18.5)$ & $18(2 \mid .2)$ & & $28(31.1)$ & $13(23.6)$ & \\
\hline TNM stage, $\mathrm{n}(\%)$ & & & 0.826 & & & 0.142 \\
\hline I-III & $61(93.8)$ & $79(92.9)$ & & $76(84.4)$ & $51(92.7)$ & \\
\hline IV & $4(6.2)$ & $6(7.1)$ & & $14(15.6)$ & $4(7.3)$ & \\
\hline Prior adjuvant chemotherapy, n (\%) & & & 0.804 & & & 0.406 \\
\hline Yes & $56(86.2)$ & $72(84.7)$ & & $72(80.0)$ & $47(85.5)$ & \\
\hline Prior adjuvant endocrine therapy, $\mathrm{n}(\%)$ & & & 0.621 & & & 0.732 \\
\hline Yes & $31(47.7)$ & $44(5 \mathrm{I} .8)$ & & $8(8.9)$ & $4(7.3)$ & \\
\hline Prior adjuvant targeted therapy, $\mathrm{n}(\%)$ & & & 0.171 & & & 0.869 \\
\hline Yes & $10(15.4)$ & $7(8.2)$ & & $14(15.6)$ & $8(14.5)$ & \\
\hline Disease-free survival interval, $n(\%)$ & & & 0.367 & & & 0.435 \\
\hline$<24$ months & $30(46.2)$ & $33(38.8)$ & & $66(73.3)$ & $37(67.3)$ & \\
\hline Metastases location, n (\%) & & & 0.426 & & & 0.268 \\
\hline Visceral & $34(52.3)$ & $50(58.8)$ & & $59(65.6)$ & $31(56.4)$ & \\
\hline Metastases number, n (\%) & & & 0.749 & & & $0.34 I$ \\
\hline Multiple & $20(30.8)$ & $37(43.5)$ & & $29(32.2)$ & $22(40.0)$ & \\
\hline First-line chemotherapy, n (\%) & & & 0.257 & & & 0.285 \\
\hline Taxane-based & $32(49.2)$ & $49(57.6)$ & & $47(52.2)$ & $33(60.0)$ & \\
\hline Vinorelbine-based & $16(24.6)$ & $12(14.2)$ & & $19(21.1)$ & $6(10.9)$ & \\
\hline Others & $17(26.2)$ & $24(28.2)$ & & $24(26.7)$ & $16(29.1)$ & \\
\hline
\end{tabular}

Abbreviations: HR, hormone receptor; IDC, invasive ductal carcinoma; ILC, invasive lobular carcinoma; MBC, metastatic breast cancer; TNM, tumor-node-metastasis.

not receiving trastuzumab therapy (median 30 months; 95\% CI: 23.333-36.667), the difference was not statistically significant $(P=0.258)$ (Figure 2C).

\section{PFS}

Two hundred and thirty-eight (80.7\%) patients showed disease progression. The median PFS for all women was 10 months (95\% CI: 9.200-10.800) (Figure 3A). The addition of trastuzumab therapy in HER2+/HR- women resulted in significantly longer PFS (median 11 months; 95\% CI: 9.363-12.637 vs no trastuzumab therapy: median 6 months;
95\% CI: 4.524-7.476) $(P=0.000)$ (Figure 3B). Significant difference in PFS was also observed between HER2+/HR+ women receiving trastuzumab therapy (median 13 months; 95\% CI: 8.676-17.324) and those not receiving trastuzumab therapy (median 10 months; 95\% CI: 8.585-11.415) ( $P=0.001$ ) (Figure 3C).

\section{Univariate and multivariate analysis}

Univariate analyses showing a trend of association for survival were entered into the Cox proportion hazard regression model, which showed that positive HR status (hazard 

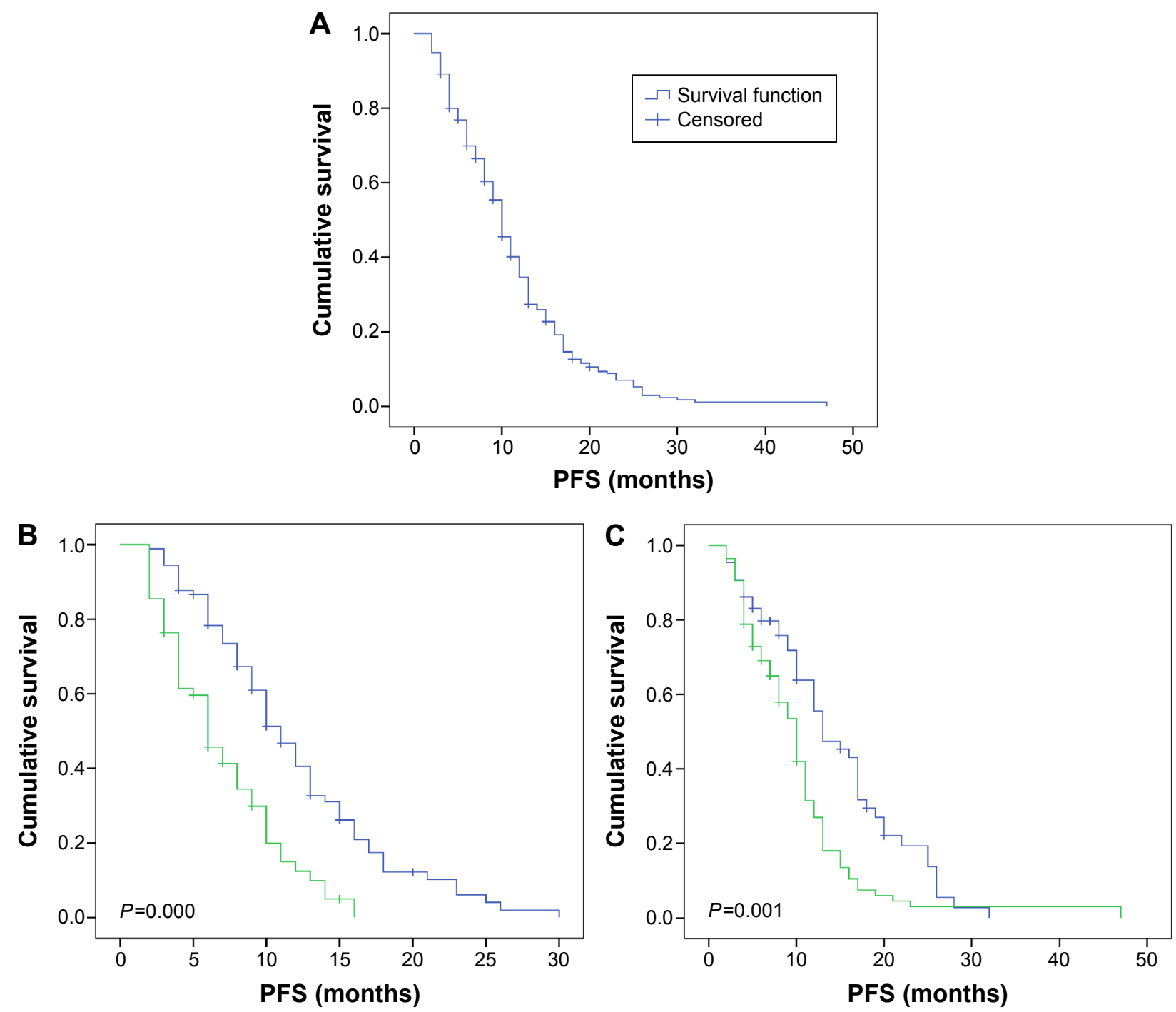

$\begin{array}{ll}\text { Treatment } & \\ \neg \text { HER2+ with trastuzumab } & + \text { HER2 + with trastuzumab-censored } \\ \neg \text { HER2 + without trastuzumab } & + \text { HER2 + without trastuzumab-censored }\end{array}$

Figure 3 Median progression-free survival (PFS) regarding HR status and trastuzumab treatment.

Notes: (A) Survival curve for all patients; (B) survival curves for HR-negative patients regarding trastuzumab treatment; and (C) survival curves for HR-positive patients regarding trastuzumab treatment.

Abbreviations: HER2, human epidermal growth factor receptor 2; HR, hormone receptor; PFS, progression-free survival.

ratio: $0.591,95 \%$ CI: $0.406-0.860, P=0.006)$ and trastuzumab therapy (hazard ratio: $0.478,95 \% \mathrm{CI}: 0.336-0.679, P=0.000$ ) were associated with statistically significant reduction in death risks and were independent predictors of OS of MBC patients (Table 3). Additionally, multivariate analysis revealed that initial TNM stage I-III (hazard ratio: 0.247, 95\% CI: $0.107-0.574, P=0.001)$ but not trastuzumab therapy (hazard ratio: $0.709,95 \% \mathrm{CI}$ : $0.436-1.154, P=0.166$ ) were associated with statistically significant reduction in death risks for HR-positive women (Table 4). Meanwhile, trastuzumab therapy (adjusted hazard ratio: 0.330, 95\% CI: 0.195-0.556,
$P=0.000$ ) were associated with statistically significant reduction in death risks for HER2+/HR- women (Table 4).

Furthermore, multivariate analysis showed that positive HR status (hazard ratio: $0.641,95 \%$ CI: $0.477-0.860$, $P=0.003$ ), non-visceral metastasis (hazard ratio: $0.702,95 \%$ CI: $0.538-0.916, P=0.009$ ), and trastuzumab therapy (hazard ratio: $0.463,95 \% \mathrm{CI}$ : $0.350-0.611, P=0.000$ ) were independent predictors of PFS of MBC patients (Table 5) and trastuzumab therapy was associated with more significant reduction in recurrence risks for HER2+/HR- women (adjusted hazard ratio: $0.369,95 \%$ CI: $0.245-0.557, P=0.000$ ) (Table 6). 
Table 3 Multivariate analysis of prognostic factors for OS in HER2-positive MBC patients

\begin{tabular}{lll}
\hline Variables & Hazard ratio (95\% Cl) & $P$-value \\
\hline Age & $1.00 I(0.985-I .018)$ & $0.89 I$ \\
TNM stage I-III vs IV & $0.668(0.388-1.149)$ & 0.145 \\
HR positivity vs negativity & $0.59 I(0.406-0.860)$ & 0.006 \\
Receiving vs not receiving prior adjuvant endocrine therapy & $1.085(0.709-1.660)$ & 0.708 \\
Disease-free survival interval $<24$ vs $\geq 24$ months & $1.288(0.880-1.885)$ & 0.193 \\
Non-visceral vs visceral metastases & $0.730(0.514-1.037)$ & 0.079 \\
Single vs multiple metastases & $0.776(0.54 I-1.115)$ & 0.170 \\
With trastuzumab in first-line treatment vs without & $0.478(0.336-0.679)$ & $0.000^{*}$ \\
\hline
\end{tabular}

Note: *Adjusted by age, initial TNM stage, HR status, prior adjuvant endocrine therapy, disease-free survival interval, metastatic location, and metastatic number.

Abbreviations: $\mathrm{Cl}$, confidence interval; HER2, human epidermal growth factor receptor 2; HR, hormone receptor; MBC, metastatic breast cancer; OS, overall survival; TNM, tumor-node-metastasis.

Table 4 Multivariate analysis of prognostic factors for OS regarding HR status of HER2-positive MBC patients

\begin{tabular}{|c|c|c|c|c|}
\hline \multirow[t]{2}{*}{ Variables } & \multicolumn{2}{|l|}{$\mathbf{H R}+$} & \multicolumn{2}{|l|}{ HR- } \\
\hline & Hazard ratio $(95 \% \mathrm{Cl})$ & $P$-value & Hazard ratio $(95 \% \mathrm{Cl})$ & $P$-value \\
\hline Age & $0.983(0.959-1.007)$ & 0.167 & $1.017(0.993-1.042)$ & 0.116 \\
\hline TNM stage I-III vs IV & $0.247(0.107-0.574)$ & 0.001 & $1.196(0.569-2.514)$ & 0.636 \\
\hline Not receiving vs receiving prior adjuvant endocrine therapy & $1.026(0.59 \mid-1.782)$ & 0.926 & I.I I $6(0.490-2.540)$ & 0.794 \\
\hline Disease-free survival interval $<24$ vs $\geq 24$ months & $1.021(0.582-1.792)$ & 0.941 & $1.660(0.966-2.852)$ & 0.067 \\
\hline Non-visceral vs visceral metastases & $0.678(0.402-1.143)$ & 0.144 & $0.694(0.423-1.140)$ & 0.150 \\
\hline Single vs multiple metastases & $0.609(0.363-1.023)$ & 0.061 & $1.080(0.640-1.824)$ & 0.773 \\
\hline With trastuzumab therapy vs without & $0.709(0.436-1.154)$ & $0.166 *$ & $0.330(0.195-0.556)$ & $0.000 *$ \\
\hline
\end{tabular}

Note: *Adjusted by age, initial TNM stage, prior adjuvant endocrine therapy, disease-free survival interval, metastatic location, and metastatic number.

Abbreviations: $\mathrm{Cl}$, confidence interval; HER2, human epidermal growth factor receptor 2; HR, hormone receptor; MBC, metastatic breast cancer; OS, overall survival; TNM, tumor-node-metastasis.

Table 5 Multivariate analysis of prognostic factors for PFS in HER2-positive MBC patients

\begin{tabular}{lll}
\hline Variables & Hazard ratio $(95 \% \mathrm{Cl})$ & $P$-value \\
\hline Age & $1.006(0.993-1.019)$ & 0.370 \\
HR positivity vs negativity & $0.641(0.477-0.860)$ & 0.003 \\
Receiving vs not receiving prior adjuvant endocrine therapy & $1.110(0.819-1.504)$ & 0.502 \\
Non-visceral vs visceral metastases & $0.702(0.538-0.916)$ & 0.009 \\
With trastuzumab in first-line treatment vs without & $0.463(0.350-0.611)$ & $0.000^{*}$ \\
\hline
\end{tabular}

Note: *Adjusted by age, HR status, prior adjuvant endocrine therapy, and metastatic location.

Abbreviations: Cl, confidence interval; HR, hormone receptor; HER2, human epidermal growth factor 2; MBC, metastatic breast cancer; PFS, progression-free survival.

Table 6 Multivariate analysis of prognostic factors for PFS regarding HR status of HER2-positive MBC patients

\begin{tabular}{|c|c|c|c|c|}
\hline \multirow[t]{2}{*}{ Variables } & \multicolumn{2}{|l|}{$\mathbf{H R}+$} & \multicolumn{2}{|l|}{ HR- } \\
\hline & Hazard ratio $(95 \% \mathrm{Cl})$ & $P$-value & Hazard ratio $(95 \% \mathrm{Cl})$ & $P$-value \\
\hline Age & $0.995(0.977-1.013)$ & 0.565 & $1.019(0.999-1.038)$ & 0.058 \\
\hline Not receiving vs receiving prior adjuvant endocrine therapy & $1.023(0.7 \mid 4-1.464)$ & 0.903 & $1.346(0.7 \mid 6-2.531)$ & 0.357 \\
\hline Non-visceral vs visceral metastases & $0.735(0.507-1.066)$ & 0.105 & $0.610(0.410-0.907)$ & 0.015 \\
\hline With trastuzumab therapy vs without & $0.556(0.38 \mathrm{I}-0.810)$ & $0.002 *$ & $0.369(0.245-0.557)$ & $0.000 *$ \\
\hline
\end{tabular}

Note: *Adjusted by age, prior adjuvant endocrine therapy, and metastatic site.

Abbreviations: $\mathrm{Cl}$, confidence interval; HR, hormone receptor; HER2, human epidermal growth factor 2; MBC, metastatic breast cancer; PFS, progression-free survival. 


\section{Discussion}

Growing evidence indicated that the response to targeted therapy and the prognostic impact of pathologic complete response (pCR) after anti-HER2-targeted therapy depended on HR status. In neoadjuvant settings, data from a retrospective study showed that the combination of trastuzumab and chemotherapy gained different clinical outcomes in HER2positive breast cancer according to HR status, and the $\mathrm{pCR}$ rates were 1.5- to 2-fold lower in ER-positive than in ERnegative patients. ${ }^{31}$ More recently, data from other clinical trials such as NeoSphere, ${ }^{32}$ NeoALTTO, ${ }^{33}$ GeparQuinto, ${ }^{34}$ NOAH, ${ }^{35}$ ACOSOG Z1041, ${ }^{36}$ and TBCRC $006^{37}$ further confirmed these findings, showing that $\mathrm{pCR}$ rates were lower in HER2+/HR+ patients, regardless of the type of anti-HER2 treatment. Moreover, a multicenter retrospective study also revealed that HR status as an independent predictor of $\mathrm{pCR}$ and trastuzumab therapy resulted in a smaller benefit in recurrence-free survival in HER2+/HR+ patients than in HER2+/HR- patients. ${ }^{38}$ Similar data were also reported in the adjuvant setting. Data from a study on HR status and the benefit of trastuzumab among MBC patients showed that high expression of ER ( $\geq 30 \%$ of tumor cells) was associated with reduced responsiveness to trastuzumab plus chemotherapy, and when endocrine therapy was added to the regimen of patients with ER higher expression tumors, significant PFS benefits were seen. ${ }^{39}$ These results suggest that the treatment strategy for HER2-positive breast cancer may be stratified by HR status.

Contrary to the situation in early breast cancer, data on the influence of HR status on trastuzumab treatment benefits in advanced HER2-positive breast cancer are limited. In our study, we evaluated the impact of HR status on the clinical outcome of trastuzumab therapy for Chinese MBC patients. Our data showed that trastuzumab administration resulted in a markedly longer OS with a 9 month increase in median OS in HER2+/HR- women compared with HER2+/ HR- women who did not receive trastuzumab therapy. This survival benefit, however, was not observed in HER2+/HR+ women. This finding is similar to previous findings from early stage and $\mathrm{MBC}$ patients. ${ }^{40,41}$ Multivariate analysis further showed that HR status was an independent predictor of OS of Chinese HER2-positive MBC patients and negative HR status was associated with statistically significant reduction in risk of death when treated with trastuzumab (hazard ratio: $0.330,95 \% \mathrm{CI}: 0.195-0.556, P=0.000$ ). In our study, HR status was also an independent predictor of PFS of MBC patients, and HER2+/HR- women had more significant reduction in progression risk. These findings suggest that
HR status modulates HER2-positive MBC patient response to trastuzumab and HR status may be used to stratify MBC patients for trastuzumab therapy. Our findings partially disagreed with the retrospective study by Brufsky et $\mathrm{al}^{42}$ who analyzed MBC patients enrolled in three clinical trials and found that the addition of trastuzumab therapy to chemotherapy significantly improved prognosis of patients with HER2-positive metastatic diseases. However, they observed no significant clinical benefit difference among HR-positive and HR-negative diseases. ${ }^{42}$ It must be noted, however, that there were some limitations in their study, such as the testing of HR status was performed in local laboratories and with potential variation. Considering the relatively lower $\mathrm{pCR}$ rate and smaller prognostic impact in HER $2+/ \mathrm{HR}+$ patients, we might reconsider less cell toxicity drugs or hormonal therapy combined with anti-HER 2 agents in this subset of population. However, in HER2+/HR- patients, chemotherapy-related toxicity therapy combined with anti-HER2 agents remain the optimal treatment.

Resistance to anti-HER2 therapy is an ongoing topic for investigation. ${ }^{43,44}$ Emerging data suggested that the bidirectional cross-talk between the HER2 and HR pathways is not only involved in endocrine resistance, but also in anti-HER2 agent resistance. Wang et al showed that, following trastuzumab and lapatinib treatment, the ER activity upregulated or dynamically transitioned and functioned as the key escape/survival pathway and led to reduced sensitivity to anti-HER2 therapy. ${ }^{44}$ Another recent preclinical study on the cross-talks between HER2 and HR pathways showed that co-expression of ER led to increased ER transcriptional activity and survivin expression, which was correlated with unfavorable response to treatment. ${ }^{45}$ Moreover, recent studies also showed that the cross-talks between phosphoinositide 3-kinase (PI3K) and the ER pathway might influence the targeted therapy efficacy. ${ }^{46} \mathrm{PI} 3 \mathrm{~K}$ mutation predicts a significant lower response with HER2+/HR+ cohort. Patients with HER 2+/HR+ tumors might be tested for PI3K mutation status, although alternative treatment options are lacking yet, but might become available in the near future. These findings partly support the clinical benefits of trastuzumab therapy in HER2+/HR - breast cancer patients and imply that dual inhibition of both HER 2 and ER achieves better responsiveness in HER2+/HR+ breast cancer patients.

The systematic treatment of HER2-positive patients enrolled in our study mostly depended on primary tumor characteristics; less than $20 \%$ of the patients received trastuzumab therapy guided by biopsy data on metastatic lesions. The conversion rate of receptor status has been reported to 
range from $10.3 \%$ to $16 \%, 30 \%$ to $40 \%$, and $5.2 \%$ to $24 \%$ for ER, PR, and HER2, respectively. ${ }^{47-50}$ Recent consensus for $\mathrm{MBC}$ recommends biopsy of metastatic lesions to re-test HER2 and HR status. ${ }^{51}$ However, the impact of receptor conversion on clinical outcome remains unclear; in a pooled analysis, only a minority of patients (14\%) changed management strategy after biopsy of metastatic lesions. ${ }^{52}$ Thus, prospective studies are required to further confirm the predictive value of the effectiveness and survival improvement based on biopsy results from metastatic lesions. Our study also has some other limitations owing to the multiple centers and retrospective nature of the study design. For example, among HER2-positive patients who received trastuzumab therapy, the duration of trastuzumab as a first-line treatment and continuation after progression varied according to economic conditions and physical performance, which might have partly impacted the results.

In People's Republic of China, a significant percentage of the HER2-positive breast cancer patients do not receive trastuzumab therapy because of drug availability and cost issues. From our data, we speculate that there is a sizable group of patients who may benefit less from the current "standard" trastuzumab and chemotherapy treatment than the rest of the patients. One of these groups is those with small tumors and high expression of both hormonal receptors in the adjuvant setting and therefore less or no chemotherapy with HER2 therapy can be considered as an option. ${ }^{53}$ On the other hand, the patients with metastatic HER2+/HR - breast cancer should be more strongly recommended to consider trastuzumab-based therapy with chemotherapy than the HER2+/HR+ group, despite the cost issues.

\section{Conclusions}

In conclusion, our study has demonstrated that HR status is an independent predictor of OS of HER2-positive MBC patients and that trastuzumab therapy significantly improves OS of HER2+/HR- patients. The predictive role of HR status on the survival benefits of anti-HER2 agents would have therapeutic implications and cause stratification by HR status of MBC patients for trastuzumab therapy, which helps to optimize cost effectiveness of trastuzumab-based regimens, especially in Chinese patients where not all people can afford expensive targeted therapy treatment.

\section{Acknowledgment}

This study was supported by the key discipline of Shijitan Hospital (2011RXZHX).

\section{Disclosure}

The authors report no conflicts of interest in this work.

\section{References}

1. Slamon DJ, Clark GM, Wong SG, Levin WJ, Ullrich A, McGuire WL. Human breast cancer: correlation of relapse and survival with amplification of the HER-2/neu oncogene. Science. 1987;235(4785):177-182.

2. Qin T, Yuan Z, Peng R, et al. HER2-positive breast cancer receiving trastuzumab treatment obtain prognosis comparable with that of HER2negative breast cancer patients. Onco Targets Ther. 2013;6:341-347.

3. Mustacchi G, Biganzoli L, Pronzato P, et al. HER2-positive metastatic breast cancer: a changing scenario. Crit Rev Oncol Hematol. 2015; 95(1):78-87.

4. Brufsky AM. Current approaches and emerging directions in HER2resistant breast cancer. Breast Cancer (Auckl). 2014;8:109-118.

5. Smith AD, Roda D, Yap TA. Strategies for modern biomarker and drug development in oncology. J Hematol Oncol. 2014;7(1):70.

6. Incorvati JA, Shah S, Mu Y, Lu J. Targeted therapy for HER2 positive breast cancer. J Hematol Oncol. 2013;6:38.

7. Romond EH, Perez EA, Bryant J, et al. Trastuzumab plus adjuvant chemotherapy for operable HER2-positive breast cancer. $N$ Engl J Med. 2005;353(16):1673-1684.

8. Piccart-Gebhart MJ, Procter M, Leyland-Jones B, et al. Trastuzumab after adjuvant chemotherapy in HER2-positive breast cancer. $N$ Engl J Med. 2005;353(16):1659-1672.

9. Slamon D, Eiermann W, Robert N, et al. Adjuvant trastuzumab in HER2positive breast cancer. $N$ Engl J Med. 2011;365(14):1273-1283.

10. Perez EA, Romond EH, Suman VJ, et al. Trastuzumab plus adjuvant chemotherapy for HER2-positive breast cancer: final planned joint analysis of overall survival (OS) from NSABP B-31 and NCCTG N9831. J Clin Oncol. 2014;32(33):3744-3752.

11. Goldhirsch A, Gelber RD, Piccart-Gebhart MJ, et al. 2 Years versus 1 year of adjuvant trastuzumab for HER2-positive breast cancer (HERA): an open-label, randomised controlled trial. Lancet. 2013;382(9897): $1021-1028$.

12. Cortes J, Saura C, Bellet M, et al. HER2 and hormone receptor-positive breast cancer - blocking the right target. Nat Rev Clin Oncol. 2011; 8(5):307-311.

13. Goldhirsch A, Ingle JN, Gelber RD, et al. Thresholds for therapies: highlights of the St Gallen International Expert Consensus on the primary therapy of early breast cancer 2009. Ann Oncol. 2009;20(8): 1319-1329.

14. Konecny G, Pauletti G, Pegram M, et al. Quantitative association between HER-2/neu and steroid hormone receptors in hormone receptor-positive primary breast cancer. J Natl Cancer Inst. 2003;95(18):142-153.

15. Lal P, Tan LK, Chen B. Correlation of HER-2 status with estrogen and progesterone receptors and histologic features in 3,655 invasive breast carcinomas. Am J Clin Pathol. 2005;123(4):541-546.

16. Untch M, Gelber RD, Jackisch C, et al. Estimating the magnitude of trastuzumab effects within patient subgroups in the HERA trial. Ann Oncol. 2008;19(6):1090-1096.

17. Sørlie T, Perou CM, Tibshirani R, et al. Gene expression patterns of breast carcinomas distinguish tumor subclasses with clinical implications. Proc Natl Acad Sci U S A. 2001;98(19):10869-10874.

18. Morrow M. Personalizing extent of breast cancer surgery according to molecular subtypes. Breast. 2013;22 (Suppl 2):S106-S109.

19. Sihto H, Lundin J, Lundin M, et al. Breast cancer biological subtypes and protein expression predict for the preferential distant metastasis sites: a nationwide cohort study. Breast Cancer Res. 2011;13(5):R87.

20. Montemurro F, Di Cosimo S, Arpino G. Human epidermal growth factor receptor 2 (HER2)-positive and hormone receptor-positive breast cancer: new insights into molecular interactions and clinical implications. Ann Oncol. 2013;24(11):2715-2724.

21. Park YH, Lee S, Cho EY, et al. Patterns of relapse and metastatic spread in HER2-overexpressing breast cancer according to estrogen receptor status. Cancer Chemother Pharmacol. 2010;66(3):507-516. 
22. Hess KR, Esteva FJ. Effect of HER2 status on distant recurrence in early stage breast cancer. Breast Cancer Res Treat. 2013;137(2):449-455.

23. Lee HJ, Park IA, Park SY, et al. Two histopathologically different diseases: hormone receptor-positive and hormone receptor-negative tumors in HER2-positive breast cancer. Breast Cancer Res Treat. 2014; 145(3):615-623.

24. Lee HJ, Seo AN, Kim EJ, et al. HER2 heterogeneity affects trastuzumab responses and survival in patients with HER2-positive metastatic breast cancer. Am J Clin Pathol. 2014;142(6):755-766.

25. Scaltriti M, Nuciforo P, Bradbury I, et al. High HER2 expression correlates with response to the combination of lapatinib and trastuzumab. Clin Cancer Res. 2014;21(3):569-576.

26. Allred DC, Carlson RW, Berry DA, et al. NCCN Task Force Report: estrogen receptor and progesterone receptor testing in breast cancer by immunohistochemistry. J Natl Compr Canc Netw. 2009;7(Suppl 6): S1-S21.

27. Hammond ME, Hayes DF, Wolff AC, et al. American Society of Clinical Oncology/College of American Pathologists guideline recommendations for immunohistochemical testing of estrogen and progesterone receptors in breast cancer. J Oncol Pract. 2010;6(4):195-197.

28. Nofech-Mozes S1, Vella ET, Dhesy-Thind S, et al. Systematic review on hormone receptor testing in breast cancer. Appl Immunohistochem Mol Morphol. 2012;20(3):214-263.

29. Wolff AC, Hammond ME, Schwartz JN, et al. American Society of Clinical Oncology/College of American Pathologists guideline recommendations for human epidermal growth factor receptor 2 testing in breast cancer. J Clin Oncol. 2007;25(1):118-145.

30. Edge SB, Compton CC. The American Joint Committee on Cancer: the 7 th edition of the AJCC cancer staging manual and the future of TNM Ann Surg Oncol. 2010;17(6):1471-1474.

31. Guarneri V, Broglio K, Kau SW, et al. Prognostic value of pathologic complete response after primary chemotherapy in relation to hormone receptor status and other factors. J Clin Oncol. 2006; 24(7):1037-1044.

32. Gianni L, Pienkowski T, Im YH, et al. Efficacy and safety of neoadjuvant pertuzumab and trastuzumab in women with locally advanced, inflammatory, or early HER2-positive breast cancer (NeoSphere): a randomized multicentre, open-label, phase 2 trial. Lancet Oncol. 2012;13:25-32.

33. Baselga J, Bradbury I, Eidtmann H, et al. Lapatinib with trastuzumab for HER2-positive early breast cancer (NeoALTTO): survival outcomes of a randomised, open-label, multicentre, phase 3 trial and their association with pathological complete response. Lancet Oncol. 2014;15(10):1137-1146.

34. Untch M, Loibl S, Bischoff J, et al. Lapatinib versus trastuzumab in combination with neoadjuvant anthracycline-taxane-based chemotherapy (GeparQuinto, GBG 44): a randomised phase 3 trial. Lancet Oncol. 2012;13(2):135-144.

35. Prat A, Bianchini G, Thomas M, et al. Research-based PAM50 subtype predictor identifies higher responses and improved survival outcomes in HER2-positive breast cancer in the NOAH study. Clin Cancer Res. 2014;20:511-521.

36. Buzdar AU, Suman VJ, Meric-Bernstam F, et al. Fluorouracil, epirubicin, and cyclophosphamide (FEC-75) followed by paclitaxel plus trastuzumab versus paclitaxel plus trastuzumab followed by FEC-75 plus trastuzumab as neoadjuvant treatment for patients with HER2positive breast cancer (Z1041): a randomised, controlled, phase 3 trial. Lancet Oncol. 2013;14(13):1317-1325.

37. Rimawi MF, Mayer IA, Forero A, et al. Multicenter phase II study of neoadjuvant lapatinib and trastuzumab with hormonal therapy and without chemotherapy in patients with human epidermal growth factor receptor 2-overexpressing breast cancer: TBCRC 006. J Clin Oncol. 2013;31:1726-1731
38. Tanioka M, Sasaki M, Shimomura A, et al. Pathologic complete response after neoadjuvant chemotherapy in HER2-overexpressing breast cancer according to hormonal receptor status. Breast. 2014;23(4):466-472.

39. Montemurro F, Rossi V, Cossu RM, et al. Hormone-receptor expression and activity of trastuzumab with chemotherapy in HER2-positive advanced breast cancer patients. Cancer. 2012;118(1):17-26.

40. Su Y, Zheng Y, Zheng W, et al. Distinct distribution and prognostic significance of molecular subtypes of breast cancer in Chinese women: a population-based cohort study. BMC Cancer. 2011;11:292.

41. Insa A, Lluch A, Prosper F, et al. Prognostic factors predicting survival from first recurrence in patients with metastatic breast cancer: analysis of 439 patients. Breast Cancer Res Treat. 1999;56(1):67-78.

42. Brufsky A, Lembersky B, Schiffman K, Lieberman G, Paton VE. Hormone receptor status does not affect the clinical benefit of trastuzumab therapy for patients with metastatic breast cancer. Clin Breast Cancer. 2005;6(3):247-252.

43. Rexer BN, Arteaga CL. Intrinsic and acquired resistance to HER2targeted therapies in HER2 gene-amplified breast cancer: mechanisms and clinical implications. Crit Rev Oncog. 2012;17(1):1-16.

44. Wang YC, Morrison G, Gillihan R, et al. Different mechanisms for resistance to trastuzumab versus lapatinib in HER2-positive breast cancers - role of estrogen receptor and HER2 reactivation. Breast Cancer Res. 2011;13(6):R121.

45. Collins DC, Cocchiglia S, Tibbitts P, et al. Growth factor receptor/ steroid receptor cross talk in trastuzumab-treated breast cancer. Oncogene. 2015;34(4):525-530.

46. Takada M, Higuchi T, Tozuka K, et al. Alterations of the genes involved in the PI3K and estrogen-receptor pathways influence outcome in human epidermal growth factor receptor 2-positive and hormone receptorpositive breast cancer patients treated with trastuzumab containing neoadjuvant chemotherapy. BMC Cancer. 2013;13:241.

47. Hoefnagel LD, van de Vijver MJ, van Slooten HJ, et al. Receptor conversion in distant breast cancer metastases. Breast Cancer Res. 2010; 12(5):R75.

48. Duchnowska R, Dziadziuszko R, Trojanowski T, et al. Conversion of epidermal growth factor receptor 2 and hormone receptor expression in breast cancer metastases to the brain. Breast Cancer Res. 2012;14(4): R119.

49. Niikura N, Liu J, Hayashi N, et al. Loss of human epidermal growth factor receptor 2 (HER2) expression in metastatic sites of HER2-overexpressing primary breast tumors. J Clin Oncol. 2012;30(6): 593-599.

50. Amir E, Miller N, Geddie W, et al. Prospective study evaluating the impact of tissue confirmation of metastatic disease in patients with breast cancer. J Clin Oncol. 2012;30(6):587-592.

51. Cardoso F, Costa A, Norton L, et al. ESO-ESMO 2nd international consensus guidelines for advanced breast cancer (ABC2). Breast. 2014; 23(5):489-502.

52. Amir E, Clemons M, Purdie CA, et al. Tissue confirmation of disease recurrence in breast cancer patients: pooled analysis of multi-center, multi-disciplinary prospective studies. Cancer Treat Rev. 2012;38(6): $708-714$.

53. Tolaney SM, Barry WT, Dang CT, et al. Adjuvant paclitaxel and trastuzumab for node-negative, HER2-positive breast cancer. $N$ Engl J Med. 2015;372(2):134-141. 


\section{Publish your work in this journal}

OncoTargets and Therapy is an international, peer-reviewed, open access journal focusing on the pathological basis of all cancers, potential targets for therapy and treatment protocols employed to improve the management of cancer patients. The journal also focuses on the impact of management programs and new therapeutic agents and protocols on

patient perspectives such as quality of life, adherence and satisfaction. The manuscript management system is completely online and includes a very quick and fair peer-review system, which is all easy to use. Visit http://www.dovepress.com/testimonials.php to read real quotes from published authors.

Submit your manuscript here: http://www.dovepress.com/oncotargets-and-therapy-journal 